\title{
A simple and efficient solution of the identifiability problem for hidden Markov sources and quantum random walks
}

\author{
Alexander Schönhuth, Member IEEE \\ Pacific Institute for the Mathematical Sciences \\ School of Computing Science \\ Simon Fraser University \\ 8888 University Drive \\ Burnaby, BC, V5A 1S6, Canada \\ schoenhuthecs.sfu.ca
}

\begin{abstract}
A solution of the identifiability problem (IP) for hidden Markov models (HMMs), based on a novel algebraic theory for random sources, is presented. It gives rise to an efficient and practical algorithm that can be easily implemented. Extant approaches are exponential in the number of hidden states and therefore only applicable to a limited degree. The algorithm can be equally applied to solve the IP for quantum random walks (QRWs) that have recently been presented as an analogon of Markov chains in quantum information theory. Moreover, the algorithm can be used to efficiently test HMMs and QRWs for ergodicity, which had remained an open problem so far.
\end{abstract}

Keywords. Hidden Markov Models, Discrete Random Sources, String Functions

\section{Introduction}

A hidden Markov model (HMM) $\mathcal{M}=(\Sigma, S, \pi, A, E)$ is specified by a finite set of output symbols $\Sigma$, a set of hidden states $S=\{1, \ldots, n\}$, a transition probability matrix $A=\left(A_{i j}\right)_{i, j \in S} \in \mathbb{R}^{n \times n}$, an initial probability distribution $\pi \in \mathbb{R}^{n}$ and an emission probability matrix $E=\left(E_{i v}\right)_{i \in S, v \in \Sigma} \in \mathbb{R}^{n \times \Sigma}$. It gives rise to a random source with values in the finite set $\Sigma$, referred to as hidden Markov source (HMS) in the following (we will use the terms HMM and HMS interchangeably if this does not lead to confusion), by the idea of changing hidden states according to the transition probabilities $A_{i j}=P(i \rightarrow j)$, where the first state is picked according to $\pi$, and emitting symbols from the hidden states, as specified by the emission probabilities $E_{i a}=P(a$ is emitted from $i)$. See e.g. [6] for a comprehensive review of the related theory.

Quantum random walks ( $Q R W s$ ) have been introduced to quantum information theory as an analogon to classical Markov sources [1]. For example, they allow to emulate Markov Chain Monte Carlo approaches on quantum computers. However, their properties are much less understood. A $\mathrm{QRW} \mathcal{Q}=\left(G, U, \psi_{0}\right)$ is specified by a directed, $K$-regular graph $G=(V, E)$, a unitary (evolution) operator $U: \mathbb{C}^{N} \rightarrow \mathbb{C}^{N}$ 
and a wave function $\psi_{0} \in \mathbb{C}^{N}$ (i.e. $\left\|\psi_{0}\right\|=1$ for $\|$.$\| the Euclidean norm) where$ $N:=K \cdot|V|=|E|$. Dimensions are labeled by edges which in turn are labeled by $(u, x)$ where $u \in V$ and $x \in X,|X|=K$ and $\mathbb{C}^{N}$ is considered to be spanned by the orthonormal basis $\left(e_{(u, x)}\right)_{(u, x) \in V \times X=E}$. Furthermore, some more specific conditions must hold that do not affect our considerations here. A QRW induces a classical random source $p_{\mathcal{Q}}$ with values in $\Sigma:=V$ (i.e. the set of nodes) by the following iterative procedure. In the first step, the evolution operator is applied to the initial wave function $\psi_{0}$, and the resulting wave function $U \psi_{0}$, with probability $\sum_{x \in X}\left|\left(U \psi_{0}\right)_{\left(u_{1}, x\right)}\right|^{2}$, is collapsed (i.e. projected and renormalized, which models a quantum mechanical measurement) to the subspace of $\mathbb{C}^{N}$, spanned by the vectors $e_{\left(u_{1}, x\right)}, x \in X$ that is associated with (the edges leaving from) node $u_{1}$, thereby generating the first symbol $u_{1}$. This procedure results in a new wave function describing the state $\psi_{u_{1}}$ the QRW is in after having generated the first symbol $u_{1}$. In order to generate a second symbol $U$ is applied to $\psi_{u_{1}}$, and $U \psi_{u_{1}}$ is, with probability $\sum_{x \in X}\left|\left(U \psi_{u_{1}}\right)_{\left(u_{2}, x\right)}\right|^{2}$, collapsed to state $\psi_{u_{1} u_{2}}$, thereby generating the second symbol $u_{2}$. Iterative application of this basic procedure of evolving followed by collapsing yields a sequence of symbols. See the subsequent subs. 2.3 for a way to formally compute the probabilities $p_{\mathcal{Q}}\left(X_{1}=v_{1}, \ldots, X_{t}=v_{t}\right)$ and also [1] for more details.

\section{Identifiability Problem (IP)}

Given two HMMs $\mathcal{M}_{1}, \mathcal{M}_{2}$ or two QRWs $\mathcal{Q}_{1}, \mathcal{Q}_{2}$, decide whether the associated random processes $p_{1}, p_{2}$ are equivalent.

For HMMs, the IP was brought up in 1957 [2] (see also [3] for a subsequent contribution). It was formulated for finite functions of Markov chains (FFMCs) that give rise to the same class of random sources as HMMs. Note that a solution of the IP for HMMs trivially applies to that of FFMCs. It was fully solved in 1992 [4]. The corresponding algorithm is exponential in the number of hidden states and therefore impractical for larger models. The formulation of the IP for QRWs is novel.

The purpose of this work is to present a simple and efficient algorithm that solves the IP for both HMMs and QRWs. Moreover, by combining the results of [7] with the presented algorithm one obtains an efficient test for ergodicity of HMMs and QRWs which, in particular for HMMs, had been a prominent open problem so far.

\section{String Functions and Prediction Matrices}

The efficiency of the algorithm is provided by a novel, vector space based approach to random sources that has been presented in [7].

Let $\Sigma^{*}=\cup_{t \geq 0} \Sigma^{t}$ be the strings of finite, but arbitrary length over $\Sigma$ where, for technical convenience, $\Sigma^{0}=\{\square\}$ with $\square$ the empty word. As justified by standard arguments, we will view random processes $p$ with values in $\Sigma$ as string functions $p$ : $\Sigma^{*} \rightarrow \mathbb{R}$ (we also write $p \in \mathbb{R}^{\Sigma^{*}}$ ) by

$$
p\left(v=v_{0} v_{1} \ldots v_{t}\right):=p\left(X_{0}=v_{o}, X_{1}=v_{1}, \ldots, X_{t}=v_{t}\right)
$$


Accordingly, it suffices to check for equality of $p_{1}(v), p_{2}(v)$ for all $v \in \Sigma^{*}$ to solve the IP for two processes $p_{1}, p_{2}$. We write $w v \in \Sigma^{s+t}$ for the concatenation of two strings $w \in \Sigma^{s}, v \in \Sigma^{t}$ and $p(v \mid w):=p(w v) / p(w)(p(v \mid w):=0$ if $p(w)=0)$ for the probability that a source $p$ emits the string $v$ after having emitted the string $w$. We then consider the infinite-dimensional matrices

$$
\mathcal{V}_{p}:=[p(w v)]_{w, v \in \Sigma^{*}} \in \mathbb{R}^{\Sigma^{*} \times \Sigma^{*}} \cong \mathbb{R}^{\mathbb{N} \times \mathbb{N}}
$$

for HMSs $p$. Furthermore, we write

$$
\begin{aligned}
f_{v}: \Sigma^{*} & \rightarrow \mathbb{R} \\
w & \mapsto p(w v)
\end{aligned} \text { resp. } \begin{aligned}
g_{w}: \Sigma^{*} & \rightarrow \mathbb{R} \\
v & \mapsto p(w v)
\end{aligned}
$$

for the row resp. column vectors of $\mathcal{V}_{p}$ which are string functions in their own right. Note that $p=g_{\square}=f_{\square}$.

Definition 1. Let $p$ be an arbitrary random source with values in $\Sigma$. Then

$$
\operatorname{dim} p:=\operatorname{rk} \mathcal{V}_{p}=\operatorname{dim} \operatorname{span}\left\{f_{v} \mid v \in \Sigma^{*}\right\}=\operatorname{dim} \operatorname{span}\left\{g_{w} \mid w \in \Sigma^{*}\right\} \in \mathbb{N} \cup\{\infty\}
$$

is referred to as dimension or minimum degree of freedom of the source $p$. If $\operatorname{dim} p<$ $\infty$ we say that $p$ is a finite-dimensional source (also referred to as a finitary or linearly dependent process in the literature).

In the following,

$$
\operatorname{span}\left\{f_{v} \mid v \in \Sigma^{*}\right\} \quad \text { resp. } \operatorname{span}\left\{g_{w} \mid w \in \Sigma^{*}\right\}
$$

will be referred to as the row resp. column space of the prediction matrix $\mathcal{V}_{p}$.

\subsection{Observable Operators}

In the following, we will introduce observable operators, acting on the vector space of string functions, and point out that rows resp. columns of the prediction matrix $\mathcal{V}_{p}$ can be obtained by applying the observable operators to $p$.

Definition 2. The linear operators

$$
\rho_{v}, \tau_{w}: \mathbb{R}^{\Sigma^{*}} \rightarrow \mathbb{R}^{\Sigma^{*}}
$$

for $v, w \in \Sigma^{*}$ that act on the vector space of string functions by means of the prescription

$$
\left(\rho_{v} f\right)(w):=f(w v) \quad \text { resp. } \quad\left(\tau_{w} g\right)(v):=g(w v)
$$

for string functions $f, g \in \mathbb{R}^{\Sigma^{*}}$ are called observable operators.

Note that

$$
\left(\rho_{v_{1}}\left(\rho_{v_{2}} f\right)\right)(w)=\left(\rho_{v_{2}} f\right)\left(w v_{1}\right)=f\left(w v_{1} v_{2}\right)=\left(\rho_{v_{1} v_{2}} f\right)(w)
$$


Hence $\rho_{v_{1} \ldots v_{t}}=\rho_{v_{1}} \circ \ldots \circ \rho_{v_{t}}$. Analogously, but in the reverse order on the letters,

$$
\tau_{w_{1} \ldots w_{s}}=\tau_{w_{s}} \circ \ldots \circ \tau_{w_{1}}
$$

By definition of $f_{v}$ and $g_{w}$,

$$
f_{v_{1} \ldots v_{t}}=\rho_{v_{1}} \circ \ldots \circ \rho_{v_{t}} p, \quad g_{w_{1} \ldots w_{s}}=\tau_{w_{s}} \circ \ldots \circ \tau_{w_{1}} p .
$$

That is, row resp. column vectors of $\mathcal{V}_{p}$ are generated by iterated application of the $\rho_{a}$ resp. $\tau_{a}$ for single letters $a \in \Sigma$ to $p$. The following lemma establishes a key observation.

Lemma 1. Let $p$ be a random source and let $\left(g_{i}\right)_{i=1, \ldots, n}$ be string functions such that the column resp. row space of $\mathcal{V}_{p}$ is contained in $\operatorname{span}\left\{g_{i}, i=1, \ldots, n\right\}$ (hence $\operatorname{dim} p \leq$ $n)$. Let $v_{0}, v_{1}, \ldots, v_{m} \in \Sigma^{*}$ such that

$$
\left(g_{1}\left(v_{0}\right), \ldots, g_{n}\left(v_{0}\right)\right) \in \operatorname{span}\left\{\left(g_{1}\left(v_{j}\right), \ldots, g_{n}\left(v_{j}\right)\right) \mid j=1, \ldots, m\right\} \subset \mathbb{R}^{n}
$$

Then it holds that

$$
\forall w \in \Sigma^{*}: \quad f_{w v_{0}} \in \operatorname{span}\left\{f_{w v_{j}} \mid j=1, \ldots, m\right\} \subset \mathbb{R}^{n}
$$

resp.

$$
\forall w \in \Sigma^{*}: \quad g_{v_{0} w} \in \operatorname{span}\left\{f_{v_{j} w} \mid j=1, \ldots, m\right\} \subset \mathbb{R}^{n}
$$

Proof. We will only prove the column space statement (12), as (13) follows from considerations that are completely analogous while respecting that here, observable operators have to be applied in the reverse order (see (9)).

We choose appropriate $\beta_{j}, j=1, \ldots, m$ such that, according to (11),

$$
\left(g_{1}\left(v_{0}\right), \ldots, g_{n}\left(v_{0}\right)\right)=\sum_{j=1}^{m} \beta_{j}\left(g_{1}\left(v_{j}\right), \ldots, g_{n}\left(v_{j}\right)\right) .
$$

Let $u \in \Sigma^{*}$. As the $g_{i}$ span the column space of $\mathcal{V}_{p}$ we find $\alpha_{i}, i=1, \ldots, n$ such that

$$
g_{u}=\sum_{i=1}^{n} \alpha_{i} g_{i}
$$

We compute

$$
\begin{gathered}
f_{v_{0}}(u)=p\left(u v_{0}\right)=g_{u}\left(v_{0}\right) \stackrel{15}{=} \sum_{i=1}^{n} \alpha_{i} g_{i}\left(v_{0}\right) \\
\stackrel{14}{=} \sum_{i=1}^{n} \alpha_{i} \sum_{j=1}^{m} \beta_{j} g_{i}\left(v_{j}\right)=\sum_{j=1}^{m} \beta_{j} \sum_{i=1}^{n} \alpha_{i} g_{i}\left(v_{j}\right) \\
\stackrel{15}{=} \sum_{j=1}^{m} \beta_{j} g_{u}\left(v_{j}\right)=\sum_{j=1}^{m} \beta_{j} p\left(u v_{j}\right)=\sum_{j=1}^{m} \beta_{j} f_{v_{j}}(u)
\end{gathered}
$$


which, as the $\beta_{j}$ had been determined independently of $u$, translates to

$$
f_{v_{0}}=\sum_{j=1}^{m} \beta_{j} f_{v_{j}}
$$

Applying the linear operator $\rho_{w}$ to 17 yields

$$
f_{v_{0} w}=\rho_{w}\left(f_{v_{0}}\right)=\sum_{j=1}^{m} \beta_{j} \rho_{w}\left(f_{v_{j}}\right)=\sum_{j=1}^{m} \beta_{j} f_{v_{j} w}
$$

which implies (12).

The lemma is crucial for the algorithm presented in the next section. In the following we will point out how to obtain the generating systems $\left(g_{i}\right)$ for HMMs and QRWs, which are needed to apply lemma 1 in the following, hence are needed for an efficient algorithm that solves the IP for HMMs and QRWs.

\subsection{Hidden Markov Models}

HMMs are well studied, see e.g. [6] for a comprehensive review of the related theory. Therefore, we restrict ourselves to recall that the probabilities $p_{\mathcal{M}}\left(v=v_{1} \ldots v_{t}\right)$ of the HMS $p_{\mathcal{M}}$ associated with the HMM, viewed as a string function as outlined above, are computed as

$$
p_{\mathcal{M}}\left(v=v_{1} \ldots v_{t}\right)=\sum_{i_{1} \ldots i_{t} \in S^{t}} \pi\left(i_{1}\right) E_{i_{1} v_{1}} A_{i_{1} i_{2}} E_{i_{2} v_{2}} \cdot \ldots \cdot A_{i_{t-1} i_{t}} E_{i_{t} v_{t}} .
$$

We obtain the following lemma that renders lemma 1 applicable to HMMs.

Lemma 2. Let $\mathcal{M}=(\Sigma, S, \pi, A, E)$ be an HMM with $n$ hidden states, that is, $|S|=n$. Let $\left(e_{i}=(0, \ldots, 0,1,0, \ldots, 0)\right)_{i=1, \ldots, n}$ be the standard basis of $\mathbb{R}^{n}$. Let $g_{i}$ be the string function defined by

$$
g_{i}\left(v=v_{1} \ldots v_{t}\right)=\sum_{i_{2} \ldots i_{t} \in S^{t-1}} E_{i v_{1}} A_{i i_{2}} E_{i_{2} v_{2}} \cdot \ldots \cdot A_{i_{t-1} i_{t}} E_{i_{t} v_{t}} .
$$

That is, $g_{i}$ is the HMS associated with the HMM $\left(\Sigma, S, e_{i}, A, E\right)$, which can be considered as a copy of $\mathcal{M}$ with the difference that the output symbol generation procedure always starts from hidden state $i$. Then it holds that the $g_{i}$ span the column space of $\mathcal{V}_{p_{\mathcal{M}}}$, the prediction matrix of the HMS $p_{\mathcal{M}}$ associated with the HMM $\mathcal{M}$.

Proof. Comparing (19) and (20) yields

$$
p_{\mathcal{M}}=\sum_{i=1}^{n} \pi(i) g_{i}
$$


Let $=w_{1} \ldots w_{s} \in \Sigma^{*}$ and $g_{w}$ the column vector in $\mathcal{V}_{p_{\mathcal{M}}}$ indexed by $w$. We have to show that

$$
g_{w}=\tau_{w} p_{\mathcal{M}} \in \operatorname{span}\left\{g_{i} \mid i=1, \ldots, n\right\} .
$$

Combining (21) with $\tau_{w} p_{\mathcal{M}}=\tau_{w_{s}} \circ \ldots \circ \tau_{w_{1}} p_{\mathcal{M}}$ reveals that it suffices to show that

$$
\tau_{w} g_{i} \in \operatorname{span}\left\{g_{i} \mid i=1, \ldots, n\right\}
$$

for a single letter $w \in \Sigma$. Therefore, we compute

$$
\begin{aligned}
& \tau_{w} g_{i}\left(v=v_{1} \ldots v_{t}\right)=g_{i}\left(w v_{1} \ldots v_{t}\right) \\
& =\sum_{i_{1} \ldots i_{t} \in S^{t}} E_{i w} A_{i i_{1}} E_{i_{1} v_{1}} A_{i_{1} i_{2}} E_{i_{2} v_{2}} \cdot \ldots \cdot A_{i_{t-1} i_{t}} E_{i_{t} v_{t}} \\
& =\sum_{j=1}^{n} E_{i w} A_{i j} \sum_{\substack{i_{2} \ldots i_{t} \\
\in S^{t-1}}} E_{j v_{1}} A j i_{2} E_{i_{2} v_{2}} \cdot \ldots \cdot A_{i_{t-1} i_{t}} E_{i_{t} v_{t}} \\
& =\sum_{j=1}^{n} E_{i w} A_{i j} g_{j}(v) .
\end{aligned}
$$

\subsection{Quantum Random Walks}

In the following let $Q^{*}$ be the adjoint of a matrix $Q \in \mathbb{C}^{m \times n}$, that is, if $Q_{i j}=a+$ $i b, a, b \in \mathbb{R}$ then $Q_{j i}^{*}=a-i b$. In the following, we consider self-adjoint matrices, that is, the set

$$
\mathcal{S}^{n}:=\left\{Q \in \mathbb{C}^{n^{2}} \mid Q=Q^{*}\right\}
$$

which is a real-valued vector space in the usual way and is usually referred to as state space in quantum mechanics. As each $Q \in \mathcal{S}^{n}$ can be written as

$$
Q=A+i B, A, B \in \mathbb{R}^{n}
$$

where $A$ is symmetric and $B$ is skew-symmetric, a basis for $\mathcal{S}^{n}$ can be composed by joining a basis of the symmetric real-valued matrices (such a basis has $n(n+1) / 2$ elements) and a basis of the skew-symmetric matrices (this has cardinality $n(n-1) / 2$ ) which reveals that $\mathcal{S}^{n}$, as a real-valued vector space, has dimension $n^{2}$.

Let $\mathcal{Q}=\left(G=(V, E), U, \psi_{0}\right)$ be a $\mathrm{QRW}$ as described in the introduction. For a node $u \in V$ let

$$
P_{u}: \mathbb{C}^{n} \longrightarrow \mathbb{C}^{n}, \psi \mapsto \sum_{(u, x), x \in X} \psi_{(u, x)} e_{(u, x)}
$$

be the projection on the subspace referring to the node $u \in V$. It can be shown, by standard quantum mechanical arguments, that, if the QRW is in state $\psi \in \mathbb{C}^{n}$ (the 
state may have been reached by iterative evolving and collapsing, as described in the introduction), the probability of emitting the symbol $u \in V$ can be computed as

$$
p_{\mathcal{Q}}(u \mid \psi)=\operatorname{tr} P_{u} U Q_{\psi}\left(P_{u} U\right)^{*}=\operatorname{tr} P_{u} U Q_{\psi} U^{*} P_{u}^{*}
$$

where $Q_{\psi}=\psi \psi^{*}$ is a self-adjoint matrix $\left(\psi \in \mathbb{C}^{n \times 1}, \psi^{*} \in \mathbb{C}^{1 \times n}\right)$ and tr is the trace, i.e. the sum of the diagonal elements of a matrix (note that these are real-valued for self-adjoint matrices). As a consequence, by induction, one can show that

$$
p_{\mathcal{Q}}\left(v=v_{1} \ldots v_{t}\right)=\operatorname{tr}\left(P_{v_{t}} U\right) \ldots\left(P_{v_{1}} U\right) Q_{\psi_{0}}\left(P_{v_{1}} U\right)^{*} \ldots\left(P_{v_{t}} U\right)^{*} .
$$

Having introduced the linear operators

$$
T_{u}: \mathcal{S}^{n} \longrightarrow \mathcal{S}^{n}, Q \mapsto\left(P_{u} U\right) Q\left(P_{u} U\right)^{*}
$$

(29) can be rewritten as

$$
p_{\mathcal{Q}}\left(v=v_{1} \ldots v_{t}\right)=\operatorname{tr} T_{v_{t}} \ldots T_{v_{1}} Q_{\psi_{0}}
$$

which illustrates that probabilities of the classical random source associated with $\mathcal{Q}$ can be computed by iterative application of linear operators and the linear trace functional. As a consequence, we obtain

Lemma 3. Let $\mathcal{Q}=\left(G=(V, E), U, \psi_{0}\right)$ be a $Q R W$ and $T_{u}, u \in V$ as given by (30). Let further $\left(Q_{i}\right)_{i=1, \ldots, n^{2}}$ be a basis of $\mathcal{S}^{n}$. Then it holds that the string functions

$$
g_{i}\left(v=v_{1} \ldots v_{t}\right):=\operatorname{tr} T_{v_{t}} \ldots T_{v_{1}} Q_{i}
$$

generate the column space of the prediction matrix $\mathcal{V}_{p_{\mathcal{Q}}}$ of the classical random source $p_{\mathcal{Q}}$, viewed as a string function, associated with $\mathcal{Q}$.

Proof. Let $w=w_{1} \ldots w_{s} \in \Sigma^{*}$ be a string over $\Sigma=V$, the set of nodes of the graph that underlies the QRW $\mathcal{Q}$. We observe that

$$
Q_{w}:=T_{w_{s}} \ldots T_{w_{1}} Q_{\psi_{0}} \in \mathbb{C}^{n^{2}}
$$

is a self-adjoint matrix and represent it over the basis $\left(Q_{i}\right)$ :

$$
Q_{w}=: \sum_{i=1}^{n^{2}} \alpha_{w, i} Q_{i} .
$$

Let $g_{w}$ be the column vector of $\mathcal{V}_{p_{\mathcal{Q}}}$ referring to $w$. We compute

$$
\begin{gathered}
g_{w}\left(v=v_{1} \ldots v_{t}\right)=p_{\mathcal{Q}}(w v) \stackrel{\text { 31 }}{=} \operatorname{tr} T_{v_{t}} \ldots T_{v_{1}} T_{w_{s}} \ldots T_{w_{1}} Q_{\psi_{0}} \\
\stackrel{(33)}{=} \operatorname{tr} T_{v_{t}} \ldots T_{v_{1}} Q_{w} \stackrel{\text { 34t }}{=} \operatorname{tr} T_{v_{t}} \ldots T_{v_{1}}\left(\sum_{i=1}^{n^{2}} \alpha_{w, i} Q_{i}\right) \\
=\sum_{i=1}^{n^{2}} \alpha_{w, i} \operatorname{tr} T_{v_{t}} \ldots T_{v_{1}} Q_{i}=\sum_{i=1}^{n^{2}} \alpha_{w, i} g_{i}(v)
\end{gathered}
$$


where the second last equation follows from the linearity of $\operatorname{tr}$ and the $T_{u}$. This translates to

$$
g_{w}=\sum_{i=1}^{n^{2}} \alpha_{w, i} g_{i}
$$

which completes the proof.

\section{The Algorithm}

\subsection{Minimal Representations}

The algorithm presented here and also the previous versions rely on the following well known result. It gives rise to a general idea for a solution of the IP. Our approach to the inherent key computation which is based on the ideas presented in section 2 will yield an efficient and also simpler algorithm.

Theorem 1 ([4]5.7]). Let $p$ be a finite-dimensional random source, that is, $d:=\operatorname{dim} p<$ $\infty$. Then there are $\pi_{0} \in \mathbb{R}^{d}$ and $T_{a} \in \mathbb{R}^{d \times d}$ for each $a \in \Sigma$ such that

$$
p\left(v=v_{0} v_{1} \ldots v_{t}\right)=\mathbf{1}_{d}^{T} T_{v_{t}} \ldots T_{v_{1}} T_{v_{0}} \pi_{0}
$$

where $\mathbf{1}_{d}=(1, \ldots, 1)^{T} \in \mathbb{R}^{d}$. The ensemble $\left(\mathbb{R}^{d},\left(T_{a}\right)_{a \in \Sigma}, \pi_{0}\right)$ is referred to as a minimal representation of $p$. Minimal representations can be computed as follows:

1. Determine words $v_{1}, \ldots, v_{d}$ and $w_{1}, \ldots, w_{d}$ such that for

$$
V:=\left[p\left(v_{i} \mid w_{j}\right)\right]_{1 \leq i, j \leq d}: \quad \operatorname{rk} V=\operatorname{dim} p
$$

As $\mathrm{rk} V=\mathrm{rk}\left[p\left(v_{i} \mid w_{j}\right)\right]=\mathrm{rk}\left[p\left(w_{j} v_{i}\right)\right]$ this implies that the $f_{v_{i}}$ resp. $g_{w_{j}}$ span the row resp. the column space of $\mathcal{V}_{p}$.

2. For each $a \in \Sigma$, compute matrices

$$
W_{a}:=\left[p\left(a v_{i} \mid w_{j}\right)\right]_{1 \leq i, j \leq d} . \in \mathbb{R}^{d \times d} .
$$

3. A minimal representation of $p$ is then given by

$$
\left(\left(V^{-1} W_{a}\right)_{a \in \Sigma}, V^{-1}\left(p\left(v_{1}\right), \ldots, p\left(v_{d}\right)\right)^{T}\right) .
$$

This yields the following general algorithm to solve the IP, given two finitedimensional processes $p_{1}, p_{2}$. Note that one can, without loss of generality, assume that output symbol sets coincide for $p_{1}, p_{2}$.

1. Determine matrices $V_{1}, V_{2}$ for $p_{1}, p_{2}$ according to (38). If $\mathrm{rk} V_{1} \neq \mathrm{rk} V_{2}$, which translates to $\operatorname{dim} p_{1} \neq \operatorname{dim} p_{2}$, output 'Not identical'.

2. If $d=\mathrm{rk} V_{1}=\mathrm{rk} V_{2}$ put

$$
V_{3}:=\left[p_{2}\left(v_{i} \mid w_{j}\right)\right]_{1 \leq i, j \leq d},
$$

that is, $V_{3}$ is the matrix for $p_{2}$ referring to the strings of $V_{1}$. If $V_{1} \neq V_{3}$, output 'Not identical'. 
3. For both processes, $p_{1}, p_{2}$, according to (39), compute matrices $W_{1 a}, W_{2 a}$ for all $a \in \Sigma$, all referring to the strings of $V_{1}$. Furthermore, determine

$$
\pi_{1} \leftarrow\left(p_{1}\left(v_{1}\right), \ldots, p\left(v_{d}\right), \pi_{2} \leftarrow\left(p_{2}\left(v_{1}\right), \ldots, p\left(v_{d}\right) .\right.\right.
$$

If $W_{1 a}=W_{2 a}$ for all $a$ as well as $\pi_{1}=\pi_{2}$, output 'Identical'. Else, output 'Not identical'.

In the last step, the positive answer is justified by that, according to (40), the minimal representations of the two HMMs are identical which implies that their processes are. Efficiency of steps 2 and 3 is immediate for HMSs and QRWs $p_{1}, p_{2}$ (for HMSs, any probabilities $p_{1}(v), p_{2}(v)$ can be computed by the forward algorithm). The idea of viewing $V$ as part of the matrix $\mathcal{V}_{p}$ and generating row and column vectors by linear operators will finally deliver an efficient subroutine to deliver matrices of the type (38) which had caused inefficiency in the extant approaches.

\subsection{Efficient Computation of $V$}

In the following, let $p$ be random source, viewed as string function, We define $f_{p}(k)$ to be the runtime necessary for computation of $p(v)$ for strings of length $k$.

Theorem 2. Let $p$ be a random source over the alphabet $\Sigma$ for which there are string functions $g_{i}, i=1, \ldots, n$ such that the conditions

$$
\operatorname{span}\left\{g_{w} \mid w \in \Sigma^{*}\right\} \subset \operatorname{span}\left\{g_{i} \mid i=1, \ldots, m\right\}
$$

or

$$
\operatorname{span}\left\{f_{v} \mid v \in \Sigma^{*}\right\} \subset \operatorname{span}\left\{g_{i} \mid i=1, \ldots, m\right\}
$$

hold. Let $d:=\operatorname{dim} p$ where $d \leq n$, due to (43]44). Then there is an algorithm of runtime $O\left(f_{p}(2 n) n|\Sigma|\right)$ that determines words $v_{i}, w_{j}, i, j=1, \ldots, d$ such that for

$$
V:=\left[p\left(v_{i} \mid w_{j}\right)\right]_{1 \leq i, j \leq d}: \quad \operatorname{rk} V=d .
$$

Proof. We only consider the case of (43), as the proof for (44) proceeds, mutatis mutandis, analogously. Consider the following algorithm.

\section{DETERMINATION OF $V$ :}

1: Define $h(v):=\left(g_{1}(v), \ldots, g_{n}(v)\right) \in \mathbb{R}^{n}$ for $g_{i}, i=1, \ldots, n$ as in lemma1

2: $A_{\text {row }} \leftarrow\{\square\}, B_{\text {row }} \leftarrow\{h(\square)\}, C_{\text {row }} \leftarrow \Sigma$.

3: while $C_{\text {row }} \neq \emptyset$ do

4: $\quad$ Choose $v \in C_{\text {row }}$.

5: $\quad$ if $h(v)$ is linearly independent of $B_{\text {row }}$ then

6: $\quad A_{\text {row }} \leftarrow A_{\text {row }} \cup\{v\}, B_{\text {row }} \leftarrow B_{\text {row }} \cup\{h(v)\}$

7: $\quad$ end if

$$
C_{\text {row }} \leftarrow C_{\text {row }} \cup\{a v \mid a \in \Sigma\}
$$

8: end while

9: Define $q(w):=\left(p(w v), v \in A_{\text {row }}\right) \in \mathbb{R}^{\left|A_{\text {row }}\right|}$. 
10: $A_{\text {col }} \leftarrow\{\square\}, B_{\text {col }} \leftarrow\{q(\square)\}, C_{\text {col }} \leftarrow \Sigma$

11: while $C_{\text {row }} \neq \emptyset$ do

12: $\quad$ Choose $v \in C_{\text {col }}$.

13: if $h(v)$ is linearly independent of $B_{\text {row }}$ then

14: $\quad A_{\text {col }} \leftarrow A_{\text {col }} \cup\{v\}, B_{\text {col }} \leftarrow B_{\text {col }} \cup\{q(w)\}$

$C_{\text {col }} \leftarrow C_{\text {col }} \cup\{v a \mid a \in \Sigma\}$

15: end if

16: end while

17: if $\left|A_{\text {row }}\right|>\left|A_{\text {col }}\right|$ then

18: Eliminate $\left|A_{\text {col }}\right|-\left|A_{\text {row }}\right|$ many strings from $A_{\text {row }}$ such that $[p(w v)]_{v \in A_{\text {row }}, w \in A_{\text {col }}}$

19: end if is regular for the remaining $v \in A_{\text {row }}$.

20: output $V:=[p(v \mid w)=p(w v) / p(w)]_{v \in A_{\text {row }}, w \in A_{\text {col }}}$.

Claim 1: During the first while loop of steps 3 to 8 , the algorithm collects strings $v$ into $A_{\text {row }}$ such that the corresponding $f_{v}$ span the row space of $\mathcal{V}_{p}$ where $|v| \leq n$ for all $v \in A_{\text {row }}$.

Proof of Claim 1: We assume the contrary, meaning that there is $v_{0} \in \Sigma^{*}$ such that $f_{v_{0}}$ is linearly independent of $\left(f_{v}\right)_{v \in A_{\text {row }}}$. Applying lemma 1 in the reverse order yields that also

$$
h\left(v_{0}\right) \notin \operatorname{span}\left\{h(v) \mid v \in A_{\text {row }}\right\} .
$$

Clearly, the algorithm can only miss a $v_{0}$ for which $h\left(v_{0}\right)$ is linearly independent in the sense of (46) if $v_{0}$ had never been collected into $C_{\text {row }}$ in step 6. This happens only in case that there is a $w_{0} \in \Sigma^{*}$ such that $w_{0}$ is a suffix of $v_{0}$ (i.e. $v_{0}=v_{1} w_{0}$ for some $\left.v_{1} \in \Sigma^{*}\right)$ and $h\left(w_{0}\right)$ had been found to be linearly dependent of $(h(v))_{v \in A_{\text {row }}}$. However, this is a contradiction to lemma 1 which states that in such a case $f_{v_{0}}$ is linearly dependent of $\left(f_{v}\right)_{v \in A_{\text {row }}}$. As $\left|A_{\text {row }}\right| \leq n$, and the algorithm does only examine strings of length $k$ that have a suffix $v_{0}$ of length $k-1$, which refers to a linearly independent $h\left(v_{0}\right)$, there are no strings of length greater than $n$ in $A_{\text {row }}$.

Claim 2: In the second while loop, strings $w$ are collected into $A_{\text {col }}$ such that $\left(g_{w}\right)_{w \in A_{c o l}}$ is a basis of the column space of $\mathcal{V}_{p}$. Moreover, $|w| \leq n$ for all $w \in A_{c o l}$.

Proof of Claim 2: As the $f_{v}, v \in A_{\text {row }}$ span the row space, one obtains, by applying lemma 1 such that the $g_{i}$ correspond to the $f_{v}$ and following the same line of argumentation as for Claim 1, that the $g_{w}, w \in A_{\text {col }}$ generate the column space. Moreover, the $g_{w}$ had been picked only if the corresponding $q(w)=(p(w v))_{v \in A_{\text {row }}}$ had been linearly independent of the $q(w)$ having been collected so far. By the definition of $\operatorname{dim} p$, there can at most be $\operatorname{dim} p$ linearly independent $q(w)$, which confirms the assertion of Claim 2. $|w| \leq n$ for all $w \in A_{\text {col }}$ follows from the considerations that are analogous to those for proving Claim 1.

As a consequence of the possible situation that $\operatorname{dim} p<\operatorname{dim} \operatorname{span}\left\{g_{i}, i=1, \ldots, n\right\}$, one might have that $\left|A_{\text {row }}\right|>\left|A_{\text {col }}\right|$. Therefore, in Step 13, the algorithm eliminates $\left|A_{\text {row }}\right|-\operatorname{dim} p$ strings from $A_{\text {row }}$ such that the remaining $f_{v}, v \in A_{\text {row }}$ still span the row space of $\mathcal{V}_{p}$. 
Due to lemma 1, vectors $h(u v)$ resp. $q(w u)$ (for arbitrary $u \in \Sigma^{*}$ ) of linearly dependent $h(v)$ resp. $q(w)$ do not have to be tested for linear dependency. Therefore, the while loops consist of at most $n \cdot|\Sigma|$ iterations, referring to the $|\Sigma|$ many continuations of each of the at most $n$ linearly independent strings.

Finally note that only probabilities $p(w v)$ for strings $w, v$ of length at most $n$ have to be calculated which gives rise to the runtime factor $f_{p}(2 n)$. This completes the proof. $\diamond$

Corollary 1. In case of p being induced by HMMs or QRWs, there is an algorithm that, efficiently in terms of the parameterizations of the HMM or QRW, determines words $v_{i}, w_{j}, i, j=1, \ldots, d$ such that for

$$
V:=\left[p\left(v_{i} \mid w_{j}\right)\right]_{1 \leq i, j \leq d}: \quad \text { rk } V=d
$$

where $d=\operatorname{dim} p$.

Proof. We obtain families of string functions $\left(g_{i}\right)_{i=1, \ldots, n}$ that span the column space of $\mathcal{V}_{p}$ from the lemmata 2 (HMMs) resp. 3 (QRWs). Application of theorem 2 then yields an algorithm of runtime $O\left(f_{p}(2 n) n|\Sigma|\right)$ where $n$ is the number of hidden states in case of HMMs and $n=|V|^{2}$, i.e. the square of the number of nodes in the graph that underlies the QRW. What remains to show is that, for both HMMs and QRWs, $f_{p}(k)$ is a polynomial in terms of the underlying parameterizations. In case of HMMs, this is guaranteed by the well known forward algorithm [6]. In case of QRWs, $f_{p}(k)$ is a polynomial in $k$ and the number of nodes of the underlying graph, as given by (29).

Corollary 2. If $p_{1}, p_{2}$ are induced by HMMs or QRWs one can determine, efficiently in terms of the underlying parameterizations, whether $p_{1}=p_{2}$ or not.

Proof. Consider the core procedure of ssec. 3.1 Efficiency of 1 is provided by cor.1. The efficiency of 2 and 3 follows from the efficiency of computing probabilities $p(v)$, as outlined in the proof of cor. 1 .

FinAl Remarks: Note that plugging the subroutine to determine $V$ into the core algorithm at the end of the page before results in a truely simple algorithm. Ergodicity of an HMM or QRW, according to [7], is equivalent to that the dimension of the eigenspace of $M:=V^{-1}\left(\sum_{a \in \Sigma} W_{a}\right)$ (see (38), (39) ) to the eigenvalue 1 is one. Hence, by means of the presented algorithm, it can be efficiently tested.

\section{References}

1. D. Aharonov, A. Ambainis, J. Kempe, U. Vazirani: "Quantum walks on graphs", Proc. of 33rd ACM STOC, New York, pp. 50-59, 2001.

2. D. Blackwell and L. Koopmans: "On the identifiability problem for functions of finite Markov chains", Annals of Mathematical Statistics, vol. 28, pp. 1011-1015, 1957.

3. E.J. Gilbert: "On the identifiability problem for functions of finite Markov chains", Annals of Mathematical Statistics, vol. 30, pp. 688-697, 1959. 
4. H. Ito, S.-I. Amari and K. Kobayashi: "Identifiability of hidden Markov information sources and their minimum degrees of freedom", IEEE Trans. Inf. Theory, vol. 38(2), pp. 324-333, 1992.

5. H. Jäger: "Observable operator models for discrete stochastic time series", Neural Computation, vol. 12(6), pp. 1371-1398, 2000.

6. Y. Ephraim, N. Merhav, "Hidden Markov processes", IEEE Trans. on Information Theory, vol. 48(6), pp. 1518-1569, 2002.

7. A. Schönhuth and H. Jäger: "Characterization of ergodic hidden Markov sources", http://www.zaik.uni-koeln.de/ paper/index.html?show=zaik2008-573, submitted to IEEE Transactions on Information Theory 